\title{
International cooperation: A brief history We've experienced
}

\author{
Wu Ji ${ }^{1,2, *}$, Bai Qingjiang ${ }^{1}$, and Xu Yongjian ${ }^{1}$ \\ ${ }^{1}$ National Space Science Center, Chinese Academy of Sciences, Zhongguancun Nanertiao 1, Haidian District, Beijing 100190, PR China \\ ${ }^{2}$ University of Chinese Academy of Sciences, Jingmibeierjie, Huairou, Beijing 101499, PR China
}

Received 30 November 2020 / Accepted 16 February 2021

\begin{abstract}
The solar-terrestrial space is of considerable significance for human activities. Since the first artificial satellite Sputnik 1 was launched in 1957, more knowledge about the dynamic conditions of the space environment has been acquired. With growing dependence on modern technology - both in space and on the ground, the vulnerability of the modern society and its infrastructure to space weather has increased dramatically. To better understand, forecast and reduce the adverse effects of space weather, science programs on space weather always prioritize the measurement or acquisition of the data from different locations of the geo-space, such as in magnetopause, polar cusps, and the magnetic tail. For the ground observations, it is necessary to locate the instruments in different longitudes and latitudes. For a single country, it is impossible to cover all these observation points. Therefore, international cooperation is very much needed. The paper reviews some of the international space weather observation programs we have experienced at the system design level. It may provide lessons learned for the community that may enable such kind of cooperative programs in the future.
\end{abstract}

Keywords: international cooperation / space weather / cooperative programs

For human being, the solar-terrestrial space, as the fourth environment after land, ocean and atmosphere, is the main domain for human space activities. In 1957, the launch of the first artificial satellite, Sputnik 1, ushered in a new era for modern space science. The following space race triggered a period of explosive growth in our knowledge of the geo-space and its interaction with the solar wind. With the development of space weather study, scientists realize that international cooperation is a must for space weather study since we need information from different locations and at different time. Multiple locations with broad spectrum sensors can never be provided by any single agency. The paper, reviewing several space weather programs we have experienced that involve international cooperation, aims to promote further international cooperation in space weather community.

\section{The first scientific satellite program of China - Double Star Program}

The previous understanding of geo-space was from a stable standard model with a magnetopause on the day side of the Earth at about $10 R_{\odot}$, a long magnetic tail on the night side, and two cusp regions over both north and south poles. However, from the 1980's, more and more scientists believed this model should be updated. It should be a dynamic one changing together with solar wind input. For a dynamic magnetosphere, it is, therefore, impossible to measure with a single satellite since one cannot determine whether the changes measured are due to the crossing of boundary or due to the time variation of the overall structure.

In the middle of 1980's, a multi-spacecraft mission called Phoenix (later changed to Cluster) was then proposed (Escoubet et al., 2001). The mission was composed of four four spacecrafts flying in a large polar orbit with Apogee of $20 R_{\odot}$ and Perigee of $4 R_{\odot}$. It was approved by the European Space Agency (ESA) and the planned launch date was mid-1996.

Since the orbit of Cluster mission is polar, there was a lack of information from the equatorial plane. Cluster team was then looking for joint measurement partners. A German small satellite mission called EQUATOR-S joined the campaign (Haerendel et al., 1999). The launch date was set just one month after Cluster.

It is known that the launch of Cluster by Ariane $\mathrm{V}$ failed. EQUATOR-S mission went into space successfully. However,

*Corresponding author.wuji@nssc.ac.cn 
it was damaged by a serious solar storm after five-month operation. When ESA decided to resume the Cluster mission in 1997, there was then no corresponding missions to cover the magnetosphere in equator region.

In order to get the maximum science output, at the beginning of the Cluster mission, ESA decided to open the data to scientists all over the world. Back then Liu Zhenxing, a Chinese professor, had an extraordinary proposal and was thereby entrusted to build the Chinese Cluster data center at the Center for Space Science and Applied Research (CSSAR) in Beijing (later renamed as National Space Science Center, or NSSC for short). In the beginning of 1997, Liu's team proposed a Chinese mission called Double Star Program (DSP). The mission objectives of DSP were to investigate physical processes of magnetospheric space storms, establish physical models of magnetospheric space storms, and to develop dynamic models and prediction techniques of the near-Earth space environment (Liu et al., 2005). It has two small s/c flying over both the polar region and the equatorial region. Prof. Wu Ji was then deeply involved into this mission at the system management level and served as the chief designer of the ground system. The Cluster Mission and Double Star Program later became a joint China-ESA cooperation.

In November 1997, R. Bonnet, the science director of ESA, led an ESA delegation to visit China. Prof. Wu Ji, then deputy director general of NSSC, Chinese Academy of Sciences (CAS), met with this delegation. At the small meeting, both sides presented their current programs. Prof. Liu then presented DSP to the ESA delegation. During the break, the ESA delegation made a quick decision to join the Chinese mission by contributing their backup instruments of the flight model of the Cluster Mission. After they presented this proposal at the second half of the meeting, the discussion was then turned to the feasibilities of this cooperation.

In the following years, NSSC tried all means internally to get funding for DSP and succeeded eventually. DSP mission was officially approved in 2001 by Commission on Science, Technology, and Industry for National Defense (COSTIND), the predecessor of Chinese National Space Administration (CNSA), and became the first scientific satellite mission in China.

For the scientific payloads of DSP, 7.5 payloads were from Europe, and 8.5 payloads were from China which include two High Energy Electron Detectors (HEED), two High Energy Proton Detectors (HEPD), two Heavy Ion Detectors (HID), one Low Energy Ion Detector (LEID), one Low Frequency Electromagnetic Wave (LFEW) Detector, as well as the Neutral Atom Imager (NAI) jointly developed by China and Europe. Teams from both sides worked together closely even during the Severe Acute Respiratory Syndrome (SARS) epidemic. The two satellites, TC-1 and TC-2, of DSP were launched in December 2003 and July 2004 respectively into equatorial and polar orbits from Xichang Satellite Launch Center and Taiyuan Satellite Launch Center, China. Together with Cluster mission, it, for first time in human history, realized the measurement of the geo-space from six points. Looking back to the implementation of DSP, we have to say, it was the mutual respect and trust between the Chinese and European team that has made the cooperation smooth and successful.
Double Star Program had been operating for 7 years and ended in 2010. At the same year, IAA awarded DSP/CLUSTER Team the IAA Laurels for Team Achievement Award. Through the six-point joint measurement, DSP has yielded great science output, e.g. the discovery of density holes in the solar wind from magnetopause, and the discovery of large scale magnetotail oscillations. Up to 2010, DSP had already produced more than 200 publications, including those published in Journal of Geophysical Research (Takada et al., 2008; Zhang et al., 2010), Annales Geophysicae (Dunlop et al., 2005; Pu et al., 2005), Geophysics Research Letters (Zhang et al., 2007; Zong et al., 2007), Chinese Journal of Geophysics (Yao et al., 2005), Chinese Science Bulletin (Lu et al., 2007), Chinese Journal of Space Science (Yao et al., 2007), etc.

\section{The China-Russia Joint Research Center on Space Weather}

The solar-terrestrial system includes the solar atmosphere, interplanetary space, magnetosphere, ionosphere, thermosphere, near space and other key regions. There is no doubt that the main research domain in the solar-terrestrial system is the Sun. In addition to the heat and light, the Sun also releases a continuous flow of particles, called solar wind. It comprises plasma and magnetic field and is of considerable significance for the planet Earth and other planets.

Apart from the space observation, ground observation is also an important way to acquire data. As China's neighbor in the north, Russia plays an important role in ground observations that covers from the Sun to the Earth's atmosphere. In 1999, CAS and Russian Academy of Sciences (RAS) Siberian Branch signed the agreement on scientific cooperation. In 2000, Prof. Wu Ji made a visit to many research institutes under the Russian Academy of Sciences, among which the Institute of Solar-Terrestrial Physics (ISTP), located in Irkustk, Siberia, was the key one. The then director general of ISTP was Academician G. Zerebtsov. They shared the same understanding of the importance of the ground observations especially in view of its distribution across the land. What's more, the Far East part of Russia has little international cooperation opportunities, making it attractive to establish a scientific relation with China, a neighboring country that has lower latitude ground observation stations. Later in November 2000, CSSAR, CAS and ISTP, RAS signed the agreement for joint studies on solar-terrestrial physics and its applications.

Both sides then decided to establish a joint research center on space weather, relying on the facilities in China and Russia, which would benefit the scientists for both countries. China provided ionosphere, solar and geomagnetic monitoring facilities, including those from NSSC, the National Astronomical Observatories of the Chinese Academy of Sciences (NAOC), the Institute of Geology and Geophysics, Chinese Academy of Sciences (IGGCAS) located in Hainan, Beijing. The Russian side provided ionosphere, solar and geomagnetic monitoring facilities, such as those located in Irkutsk, Yakutsk, Lovsk, and Zhigansk. Most of the facilities are still in operation now, from which data are being acquired. The joint research covers 
the surface of the Sun, the interplanetary space, magnetosphere, ionosphere, and Middle and Upper atmosphere.

Joint decision was also made to establish a fast paper award to award the team that publishes the first joint research paper under this mechanism. Joint workshops were organized in both countries every year with meeting venues alternating between the two countries. After 2010, it was decided to have it every 2 years instead of every year, and 14 workshops had been organized by 2018 , with the 15 th workshop in planning. The research teams have made outstanding progress in various aspects such as classification of ionospheric irregularities, the comparative research on ionosphere over different latitudes, disturbance and propagation of ionosphere, and have provided important observation data for the improvement of International Reference Ionosphere (IRI). There were already over 200 scientific papers published including those on Journal of Geophysical Research (Shi et al., 2011), Annales Geophysicae (Wang et al., 2010), Plasma Science and Technology (Shi et al., 2014), Advanced Space Research (Ratovsky et al., 2014; Wang et al., 2017), Solar-Terrestrial Physics (Wang et al., 2017), Chinese Journal of Space Science (Romanova et al., 2013; Ratovsky et al., 2014), etc., and more than 40 scientific projects were implemented within the framework of the joint research center.

\section{China-Russia Joint Mission on Mars Exploration - Yinghuo-1}

The safety of spacecraft cruising in the interplanetary space is also at stake when they are exposed to space weather events, e.g. Interplanetary Coronal Mass Ejections (ICMEs). The orbiters and landers that were sent to the Venus, Mars and various asteroids, can also be affected by solar eruptions. Therefore, "space weather" is a concept extending to the entire solar system, instead of only confining to the geospace.

Yinghuo-1 (YH-1) was a joint mission between China and Russia. The s/c of YH-1 was developed by CNSA. Yinghuo-1 orbiter principally aimed to detect Martian space magnetosphere, ionosphere, plasma distribution, ions escape mechanism (Zheng et al., 2013). It was piggybacked by Russia's PhobosGrunt (FGSC) in November 2011. Joint measurements of Martian space environment by $\mathrm{YH}-1$ and Phobos-Grunt were planned. The scientific objectives were,

1. To study in details Martian space magnetosphere, ionosphere and the plasma distribution and its variations.

2. To study Martian ions escape mechanism.

3. To conduct a moderate survey of Martian surface topography and sandstorms.

4. To explore the Martian gravity field near equator by using ground VLBI network.

Four scientific payloads on-board were Plasma Detector package, Fluxgate magnetometer, Occultation Receiver and Optical Imager, i.e., a visible light camera. Among them, the plasma detector package consisted of two ion analyzers, one electron analyzer and one electronics box. The ion analyzers were to measure the energy, angle and composition of ions, while the electron analyzer detects the energy and angle of electrons. Fluxgate magnetometer consisted of two detectors and one electronics box, to measure the magnetic field in the Martian space environment (Wu et al., 2010).

This mission was triggered by an email from the Space Research Institute (IKI) of the Russian Academy of Sciences to NSSC. IKI was planning to resume the Mars mission and asked whether China wants to take this opportunity to piggyback one small scientific satellite into geo-space, but not to the Mars. After a short internal discussion, the preliminary decision has been made to go to the Mars, although Chinese spacecraft had never been there before.

About the scientific objectives, decision has been made to measure the Mars space environment, or the Mars space weather, since it has never been measured with both magneto field detectors and plasma detectors before. Another unique or first time opportunity was that with two spacecrafts, ionosphere occultation measurement could be realized to measure the profile of the ionosphere in particular at the noon and midnight local time when the extreme conditions occur.

After strenuous application process for the mission funding, the mission was approved by CNSA in 2007 and Prof. Wu Ji was appointed as the Chief Scientist in charge of the application system as well as the science payload development.

Lavochkin Association and IKI developed the FGSC; Shanghai Academy of Spaceflight Technology and NSSC, jointly developed the YH-1 mission (Ping et al., 2010). The spacecraft was launched on 8 November 2011 from the Baikonur Cosmodrome in Kazakhstan into an elliptical Earth orbit. YH-1 was supposed to be separated from Phobos-Grunt once it gets into Mars' gravitational field, then enter into a large elliptical orbit around the Mars. It was a pity that the departure engine was not ignited and both Phobos-Grunt and YH-1 failed to go to the Mars. They were both stuck in the low earth orbit and re-entered the Earth atmosphere after two months.

The Chinese satellite missions and observation facilities, represented by the above-mentioned DSP, China-Russia joint center, and Yinghuo-1, greatly contribute to space weather studies. They promoted further integration of scientific innovations and social needs, and were conducive to the establishment of the global space weather forecast framework with even greater scientific and social benefits. However, the list was far from finished, and more missions are needed to address the puzzles of space weather. The missions under development focus mainly on the Sun and solar activity phenomena, such as flares and CMEs (Gan et al., 2015), while Solar wind Magnetosphere Ionosphere Link Explorer (SMILE) mission aims to explore the sun-earth connection in a bid to set a new model for international cooperation.

\section{Solar wind Magnetosphere lonosphere Link Explorer (SMILE)}

SMILE is a joint mission between ESA and CAS to study the interaction between the Earth's magnetosphere and the solar wind, while simultaneously monitoring the magnetosphere's plasma environment (Millan et al., 2019).

It was the result of the joint effort to explore new models of cooperation between CAS and ESA since the Double Star Program. The idea of an joint mission that starts from mission 
definition could be traced back before the 9th ESA-China Space Science Bilateral Meeting held in May 2013 where ESA and CAS officially entered the agreement to start a brand new joint mission. The idea was jointly formulated by the former ESA science director A. Gimenez and Prof. Wu Ji after intensive discussions on how to continue the bilateral cooperation, and the bottom-up approach for mission selection was chosen, a common practice for both ESA and China when it comes to scientific mission selection.

The joint mission was the second CAS-ESA space science collaboration at the mission level. To prepare the release of the joint call, two joint workshops were organized, with the first CAS-ESA Joint Scientific Space Mission Workshop held in Chengdu in February 2014 and the second workshop held in Copenhagen in September 2014.

The Chengdu workshop attracted 98 scientists coming from 11 countries, who delivered 4 invited talks, 27 oral presentations, among which 12 from Europe and 15 from China, and 18 posters among which 14 from Europe and 4 from China. During the Chengdu workshop, the proposals were coming from teams with only Chinese scientists or teams with only European scientists, while during the Copenhagen workshop, only the proposals that made by a joint Chinese-European team were accepted. After the Copenhagen workshop, an official joint call for proposals was issued. It turned out that 13 joint proposals were received. After the technical feasibility screening made by a joint technical team, there were four of them left in the short list. A joint selection committee was set up to review these four proposals, out of which SMILE was finally selected. The SMILE mission was officially selected as the ESA-China joint scientific mission at the 11th ESA-China Space Science Bilateral Meeting held in Cordoba, Spain on May 20, 2015, after which both sides started the process for mission adoption by respective decision-making bodies.

Selected due to its innovative novelty and scientific ingenuity, SMILE will investigate the dynamic response of the Earth's magnetosphere to the solar wind in a unique manner, an attempt never tried before: it will combine soft X-ray imaging of the Earth's magnetopause and magnetospheric cusps with simultaneous UV imaging of the Northern aurora. SMILE will, for the first time, be able to trace and link the processes of solar wind injection in the magnetosphere with the charged particles precipitating into the cusps and eventually causing the aurora. SMILE will also carry in-situ instrumentation to monitor the solar wind and magneto-sheath plasma conditions, so that the simultaneous X-ray and UV-images can be compared and contrasted directly to reveal the upstream and local driving conditions in self-sufficient manner.

The key science questions for SMILE are:

1. What are the fundamental models of the dayside solar wind/magnetosphere interaction?

2. What defines the sub-storm cycle? and

3. How do CME-driven storms arise and what is their relationship to sub-storms?

The payloads of SMILE consist of four instruments, namely SXI (Soft X-ray Imager), UVI (UV Imager), LIA (Light Ion Analyzer) and MAG (Magnetometer). SXI is a telescope with a wide field of view microchannel plate optic and CCD detector at the focal plane. UVI is a wide field of view optic sensitive to the Lyman-Birge-Hopffman band of ultraviolet radiation. LIA is a wide field of view proton and alpha particle analyzer. MAG is a dual-redundant digital fluxgate magnetometer, with two tri-axial fluxgate sensors connected by a boom to a spacecraftmounted electronics box.

The no-fund-exchange principle was reached on the bilateral meeting. In terms of labor distribution, CAS is responsible for the study and development of satellite Platform, TC/TM (CLTC), Science Application System as well as Ground Support System, and provides in-situ measurement instruments MAG and LIA, while ESA is responsible for the study and development of Payload Module, Launch Vehicle, Launch Site, and science operation and ground receiving station supports when necessary, and also the development of the SXI and UVI (Wang \& Branduardi-Raymont, 2020).

SMILE was eventually approved by the CAS in 2016 and adopted by ESA in 2019. As an international cooperation mission dedicated to space weather research, SMILE also becomes the second S-Class mission for ESA after CHEOPS (CHaracterising ExOPlanets Satellite) which was launched in December 2019. In January 2020, SMILE passed the joint Mission Preliminary Design Review (PDR) and is scheduled to be launched from the Kourou launch site in 2023. However, the decision to postpone the launch date to 2024 was made on the 16th China-ESA Space Science Bilateral Meeting held on December 1, 2020 due to "a series of negative factors", e.g. the ongoing COVID-19 pandemic and export control policies. At present, both ESA and China are working around the clock to strengthen cooperation and coordination to overcome the delays caused by those discouraging factors, hoping it will bring long-term benefits to the future cooperation at the mission level.

As evidenced by the selection of SMILE mission, ChinaESA Space Science Bilateral Meeting, jointly initiated by ESA and CAS in 2004, played a crucial role for in-depth communications which was conducive to bilateral cooperation at payload level and mission level at large.

\section{Summary}

The human being has entered the space age for 60 years and had great advancements in understanding the space environment, which turned out to be dynamic and dangerous, rather than empty and quiet. It affects the performance and reliability of technological systems and endangers human life and health. Space weather forecast, the forecast of its source and the forecast of the solar storms in particular, is still a scientific frontier to be addressed.

Due to its massive scale, the dynamic property and time dependence while the earth is rotating, the space weather observation from the Sun all the way to the earth magnetic field is very complicated and, therefore, international cooperation is necessary which leads to data acquisition from different geo-locations, at different local time and even different regions in geo-space.

International cooperation depends strongly on the mutual trust between the scientists and should be supported by the government. In general, it needs a bottom-up process, i.e. to be proposed from the research teams. Since the final output of science will be made by the scientists, the bottom-up process is the only 
way to maintain their interests and enthusiasm. However, since the research fund is always from the government agencies, it is also important to explain clearly the significance of the project to the government agencies. The bottom-up process and the government support are the two key elements of any international programs for space weather research and observations.

Space weather is not only a basic research issue. We have already experienced several severe space weather events in the space age. The Sun has existed for 4.6 billion years, but only six solar cycles have been observed after we enter the space age. The solar eruption may lead to more severe solar storms and cause enormous adverse impacts on the Earth than we can imagine. Therefore, to study it and give warning in advance to protect our society is a very important job and duty for the space community. Unlike the terrestrial weather, space weather is a global issue. Countries around the globe must work together to foster international collaborations and prepare for the extreme space weather events in the future.

\section{References}

Dunlop MW, Taylor MGG, Davis JA, Pu ZY, Fazakeley AN, et al. 2005. Comparative Cluster/Double Star observations of the high and low latitude dayside magnetopause. Ann Geophys 2867-2875. https://doi.org/10.1.1.694.8654.

Escoubet CP, Fehringer M, Goldstein M. 2001. The Cluster Mission. Ann Geophys 19: 1197-1200. https://doi.org/10.5194/angeo-191197-2001.

Gan WQ, Geng YY, Li H, Wu J, Zhang HY, et al. 2015. ASO-S: Advanced Space-based Solar Observatory. Proc SPIE - Int Soc Opt Eng 9604: 96040T. https://doi.org/10.1117/12.2189062.

Haerendel G, Torbert RB, Höfner H. 1999. The Equator-S Mission. Ann Geophys 17: 1499-1502. https://doi.org/10.1007/s00585-9991499-x.

Liu ZX, Escoubet P, Cao JB. 2005. A Chinese-European Multiscale Mission: The Double Star Program. Multiscale Coupling of Sun-Earth Processes, pp. 509-514. https://doi.org/10.1016/B978-044451881-1/ 50037-X.

Lu L, McKenna-Lawlor S, Barabash S. 2007. Plasma sheet stretching accompanied by field aligned energetic ion fluxes observed by the NUADU instrument aboard TC-2. Chin Sci Bull 52: 1719-1723. https://doi.org/10.1007/s11434-007-0238-5.

Millan RM, Steiger VR, Ariel M, Bartalev S, Borgeaud M, et al. 2019. Small satellites for space science A COSPAR scientific roadmap. Adv Space Res 64: 1466-1517. https://doi.org/10.1016/j. asr.2019.07.035.

Ping JS, Qian ZH, Hong XY, Zheng WM, Fung LW, et al. 2010. Brief introduction about Chinese Martian Mission Yinghuo-1. In: 41st Lunar and Planetary Science Conference. $1060 \mathrm{p}$.

Pu ZY, Xiao CJ, Zhang XG, Huang ZY, Fu SY, et al. 2005. Double Star TC-1 observations of component reconnection at the dayside magnetopause: A preliminary study. Ann Geophys 23: 2889-2895. https://doi.org/10.5194/angeo-23-2889-2005.

Ratovsky KG, Shi JK, Oinats AV. 2014a. Comparative study of high-latitude, mid-latitude and low-latitude ionosphere on basis of local empirical models. Adv Space Res 54: 509-516. https://doi. org/10.1016/j.asr.2014.02.019.
Ratovsky KG, Shi JK, Oinats AV. 2014b. Comparison of diurnal, seasonal and solar cycle variations of high-latitude, mid-latitude and low-latitude ionosphere. Chin J Space Sci 34: 143-153. https://doi.org/10.11728/cjss.2014.02.143.

Romanova EB, Zherebtsov GA, Ratovsky KG. 2013. Response of the ionospheric $\mathrm{F}_{2}$-region over Irkutsk and Hainan to strong geomagnetic storms. Chin J Space Sci 33: 494-500. https://doi. org/10.11728/cjss2013.05.494.

Shi JK, Wang GJ, Reinisch BW. 2011. The relationship between strong range spread $\mathrm{F}$ and ionospheric scintillations observed in Hainan from 2003 to 2007. J Geophys Res 116: A08306. https://doi.org/10.1029/2011JA016806.

Shi JK, Wang Z, Tao W. 2014. Investigation of total absorption of radio waves in high latitude ionosphere. Plasma Sci Technol 16: 833-836. https://doi.org/10.1088/1009-0630/16/9/05.

Takada T, Nakamura R, Juusola L, Amm O, Baumjohann W, et al. 2008. Local field-aligned currents in the magnetotail and ionosphere as observed by a Cluster, Double Star, and MIRACLE conjunction. J Geophys Res 113: A07S20. https://doi.org/10.1029/ 2007JA012759.

Wang C, Branduardi-Raymont G. 2020. Update on the ESA-CAS Joint Solar Wind Magnetosphere Ionosphere Link Explorer (SMILE) Mission. Chin J Space Sci 40: 700-703. https://doi. org/10.11728/cjss2020.05.700.

Wang GJ, Shi JK, Wang X. 2010. The statistical properties of spread $F$ observed at Hainan station during the declining period of the 23rd solar cycle. Ann Geophys 28: 1263-1271. https://doi.org/ 10.5194/angeo-28-1263-2010.

Wang GJ, Shi JK, Wang Z. 2017a. Solar cycle variation of ionospheric parameters over the low latitude station Hainan, China, during 2002-2012 and its comparison with IRI -2012 model. Adv Space Res 60: 381-395. https://doi.org/10.1016/j. asr.2016.12.013.

Wang Z, Shi JK, Wang GJ. 2017b. Diurnal, seasonal, annual, and semiannual variations of ionospheric parameters at different latitudes in East Asian sector during ascending phase of solar activity. Sol-Terr Phys 3: 43-50. https://doi.org/10.12737/stp-3220177.

Wu J, Zhu GW, Zhao H. 2010. Scientific objectives of China-Russia Joint Mars exploration program YH-1. Chin Astron Astrophys 34: 163-173. https://doi.org/10.1016/j.chinastron.2010.04.009.

Yao L, Liu SL, Jin SP. 2005. A study of orientation and motion of flux transfer events observed on the high-latitude dayside magnetopause. Chin J Geophys 48: 1217-1225.

Yao L, Liu SL, Liu K. 2007. Joint Study on Transfer Events Using the Data Observed by TC-1 and Cluster Near Dayside Magetospause. Chin J Space Sci 27: 13-18. https://doi.org/10.11728/ cjss2007.01.013.

Zhang LQ, Liu ZX, Ma ZW. 2010. X Line distribution determined from Earthward and tailward convective bursty flows in the central plasma sheet. J Geophys Res 115: A06218. https://doi.org/ 10.1029/2009JA014429.

Zhang HZ, Pu ZY, Cao X, Fu SY, Liu ZX, et al. 2007. TC-1 observations of flux pileup and dipolarization-associated expansion in the near-Earth magnetotail during substorms. Geophys Res Lett 34: L03104. https://doi.org/10.1029/2006GL028326s.

Zong QG, Zhou XZ, Li X, Song P, Fu SY, et al. 2007. Ultralow frequency modulation of energetic particles in the dayside magnetosphere. Geophys Res Lett 34: L12105. https://doi.org/ 10.1029/2007GL029915. 